Disclosure of Interests: None declared

DOI: 10.1136/annrheumdis-2019-eular.4683

\section{SAT0197 INCIDENCE AND OUTCOME OF NON-TYPHOID SALMONELLA BACTEREMIA IN PATIENTS WITH SYSTEMIC LUPUS ERYTHEMATOSUS IN COMPARISON WITH RHEUMATOID ARTHRITISAND PRIMARY SJÖGREN'S SYNDROME}

Cheng-Hsun Lu ${ }^{1,2}$, LI Ko-Jen ${ }^{1}$, Cheng-Han $\mathrm{Wu}^{1}{ }^{1,2}$, Song-Chou Hsieh ${ }^{1} .{ }^{1}$ National Taiwan University Hospital, Division of Allergy, Immunology and Rheumatology, Department of Internal Medicine, Taipei, Taiwan, Republic of China; ${ }^{2}$ National Taiwan University, Graduate Institute of Clinical Medicine, College of Medicine, Taipei, Taiwan, Republic of China

Background: Non-typhoid Salmonella (NTS) infection is an important problem in patients with systemic lupus erythematosus (SLE) [1, 2]. However, less is known about the incidence rate (IR) for NTS bacteremia.

Objectives: To evaluate the IR of NTS bacteremia in patients with SLE, in comparison to rheumatoid arthritis (RA) and primary Sjögren's syndrome (SjS), and by comparison with other Gram-negative bacilli (GNB) bacteremia including Escherichia coli (E. coli) and Pseudomonas aeruginosa (PsA). Furthermore, to access the survival outcome after NTS bacteremia. Methods: In this single-center retrospective cohort study, we analyzed the registry of catastrophic illnesses database for patients fulfilled the criteria of SLE, RA, and SjS in National Taiwan University Hospital. The patients aged over 20 years, with adequate follow-up during January 1, 2008 to December 31, 2017 were included. Only new episodes of positive blood cultures during the observation period were taken into analysis. Cox proportional hazards regression model was applied for calculating the incidence rate ratio (IRR) of bacteremia between different diseases, and the hazard ratio $(\mathrm{HR})$ of mortality.

Results: This study included 5953 patients. NTS bacteremia happened in 33 cases, among which $27(81.8 \%)$ were group D1 and $4(12.1 \%)$ were group B Salmonella. The baseline characteristics, IR of each GNB bacteremia, and the IRR adjusted for age, sex, diabetes mellitus (DM) as well as chronic kidney disease (CKD) are shown in Table 1. Compared to patients with RA, the IRR for NTS bacteremia was highest in SLE. The IRR for E. coli bacteremia in patients with SLE also increased in comparison with RA, although not as high as for NTS bacteremia. When compared to SjS, patients with SLE still had an elevated IRR of 3.52 (95\% Cl: $1.29-9.63, p=0.014)$ for NTS bacteremia. In SLE patients, DM increased the IR for NTS bacteremia (IRR: $4.43,95 \% \mathrm{Cl}$ : $1.71-11.43$, $p=0.002$; adjusted for age and sex).

In the 33 cases with NTS bacteremia [male: 21.2\%; age: 49.0 (37.964.2) years], the median observation time to event was 11.1 (IQR, 3.038.1) months. No recurrent bacteremia was found. There were 14 $(42.4 \%)$ deaths, and $7(21.2 \%)$ died within 60 days after NTS bacteremia. The median time to all-cause mortality was 54 (IQR, 17-153) days. In survival analysis, an abnormal body mass index (BMI, normal $>18.5$ and $<24)$ at the onset of bacteremia had a crude HR of $6.20(95 \% \mathrm{Cl}$ : 1.64-23.52, $p=0.007)$. The diagnosis of SLE, age, gender, DM, CKD, levels of C-reactive protein and white blood cell count did not significantly increase HR.

\begin{tabular}{|c|c|c|c|}
\hline Table 1 & & & \\
\hline \multirow{2}{*}{ Diagnosis } & SLE & SjS & RA \\
\hline & $n=2057$ & $n=1532$ & $n=2364$ \\
\hline Age (y) [median (IQR)] & $40.0(30.2-52.5)$ & $56.3(47.4-64.3)$ & $56.7(47.7-66.4)$ \\
\hline Male gender (\%) & 11.3 & 9.1 & 18.3 \\
\hline $\begin{array}{l}\text { Observation time (y) } \\
\text { [median (IQR)] }\end{array}$ & $3.22(0.81-7.34)$ & $1.75(0.46-4.2)$ & $2.77(0.66-6.96)$ \\
\hline NTS bacteremia (n) & 26 & 5 & 2 \\
\hline IR per $1000 \mathrm{PY}(95 \% \mathrm{Cl})$ & $3.10(2.05-4.44)$ & $1.18(0.42-2.54)$ & $0.22(0.04-0.67)$ \\
\hline Adjusted IRR (95\% CI) & $19.02(4.35-83.24)^{* *}$ & $5.72(1.08-30.17)^{*}$ & Reference \\
\hline E. coli bacteremia $(n)$ & 61 & 15 & 34 \\
\hline IR per $1000 \mathrm{PY}(95 \% \mathrm{CI})$ & $7.26(5.59-9.24)$ & $3.54(2.03-5.64)$ & $3.71(2.60-5.11)$ \\
\hline Adjusted IRR ( $95 \% \mathrm{CI}$ ) & $2.63(1.67-4.16)^{* *}$ & $0.83(0.45-1.54)$ & Reference \\
\hline PsA bacteremia (n) & 14 & 4 & 5 \\
\hline IR per 1000 PY $(95 \% \mathrm{Cl})$ & $1.66(0.93-2.68)$ & $0.94(0.29-2.19)$ & $0.54(0.19-1.17)$ \\
\hline Adjusted IRR ( $95 \%$ CI) & $3.03(0.98-9.35)$ & $1.80(0.47-6.94)$ & Reference \\
\hline
\end{tabular}

Conclusion: Among the GNB bacteremia, patients with SLE were especially susceptible to NTS bacteremia when compared to RA and SjS. DM was a risk factor. The mortality rate after NTS bacteremia was high, and an abnormal BMI may predict it.

\section{REFERENCES}

[1] Green L, Vinker S. Recurrent salmonella sepsis with different species in a systemic lupus erythematosus patient. Clin Rheumatol. 1996 Jan; 15 (1):72-74

[2] Huang CF, Chen PL, Liu MF, Lee CC, Lee NY, Chang CM, et al. Nontyphoidal Salmonella bacteremia in patients with connective tissue diseases. J Microbiol Immunol Infect. 2012 Oct; 45(5):350-355.

Disclosure of Interests: None declared DOI: 10.1136/annrheumdis-2019-eular.5621

\section{SAT0198 PERIPHERAL NERVOUS SYSTEM INVOLVEMENT IN PRIMARY SJOGREN'S SYNDROME - UNCOMMON OR UNDERESTIMATED PROBLEM?}

Marta Jaskolska, Magdalena Chylinska, Anna Masiak, Zenobia Czuszynska, Katarzyna Galecka, Mariusz Sieminski, Żaneta Smoleńska, Marcin Ziętkiewicz Zbigniew Zdrojewski. Medical University of Gdansk, Department of Internal Medicine, Connective Tissue Diseases and Geriatrics, Gdansk, Poland

Background: Systemic manifestations are common in primary Sjögren's syndrome (pSS) and play a major role in the prognosis. Neurological complications may affect both the peripheral (PNS) and central nervous system (CNS). The incidence varies from several to several dozen percent. The course of the disease and the severity of the symptoms may be mild and self-limiting or progressive, leading to permanent neurological deficits. It is worth remembering that the symptoms of nervous system involvement may precede the onset of symptoms of dryness, and diagnosis of pSS may be delayed after a certain duration of neurological symptoms.

Objectives: The aim of the study was to determine the prevalence of PNS involvement among symptomatic and asymptomatic patients with the diagnosis of pSS in our University Clinical Centre.

Methods: We studied a consecutive group of fifty unselected patients aged from 33 to 74 years (mean 55.8 years). All patients fulfilled the criteria for the diagnosis of pSS. Additional connective tissue diseases and diabetes were the exclusion criteria. Disease activity was evaluated according to the EULAR Sjögren's syndrome disease activity index (ESS DAI). All patients underwent clinical neurological examination and nerve conduction studies (NCS) of nine peripheral nerves. For the classification of polyneuropathies the ESTEEM (European Standardized Telematic tool to Evaluate Electrodiagnostic Methods) guidelines were used. Clinical examination and nerve conduction study were performed and evaluated by one certified neurologist.

Results: Of our 50 patients, 48 were female with a mean $( \pm S D)$ age $53.6 \pm 10.5$ years and mean disease duration $7.9 \pm 5.3$ years. Two were male with mean age $43.7 \pm 25.8$ years and mean disease duration $5.7 \pm 0.6$ years. The mean age at diagnosis was $50.4 \pm 14$ years. $23(46 \%)$ patients fulfilled the criteria for the diagnosis of neuropathy. The most common PNS manifestation was sensorimotor neuropathy $11 / 23$ (47\%), mononeuropathy was present in $6 / 23(26 \%)$ patients, pure axonal sensory neuropathy was present in $1 / 23(4.3 \%)$ patient, axonal motor neuropathy in $1 / 23$ $(4.3 \%)$, SFN in $1 / 23(4.3 \%)$ and cranial nerve involvement was present in $4 / 23(17.4 \%$ ) (one of the patients had both cranial and sensorimotor neuropathy). Neurological symptoms preceded the diagnosis of pSS in 8 $(35 \%)$ of 23 PNS+ patients. The frequency of following symptoms and extraglandular manifestations was significantly higher in PNS+ compared to PNS- patients: salivary gland enlargement (74\% vs $44 \% \mathrm{p}<0,05)$, respiratory tract involvement $(65 \%$ vs $37 \% \quad \mathrm{p}<0,05)$ and lymphadenopathy $(61 \%$ vs $19 \% \mathrm{p}<0,05)$. The mean ESSDAI in patients with and without neurological involvement was $7 \pm 6,82$ and $4,7 \pm 4,58$ respectively $(p=0.245)$. In this subgroup the use of cyclophosphamide, due to extraglandular manifestations, was increased $(p<0,05)$.

Conclusion: We found that PNS involvement is a common extraglandular manifestation of pSS ( $46 \%$ in our group). Sensorimotor neuropathies were most frequent. Involvement of the PNS seems frequent but remains underestimated. NCS is a non-invasive test which might be useful at diagnosis and follow-up. Guidelines for the diagnosis and treatment of peripheral neuropathies in patients with pSS are needed.

Disclosure of Interests: None declared

DOI: 10.1136/annrheumdis-2019-eular.4851 